\title{
OPEN Sustainable production of camptothecin from an Alternaria sp. isolated from Nothapodytes nimmoniana
}

\author{
I. A. H. Khwajah Mohinudeen ${ }^{1}$, Rahul Kanumuri ${ }^{1}$, K. N. Soujanya ${ }^{2,3}$, R. Uma Shaanker ${ }^{2}$, \\ Suresh Kumar Rayala ${ }^{1}$ \& Smita Srivastava ${ }^{1 \bowtie}$
}

Camptothecin the third most in demand alkaloid, is commercially extracted in India from the endangered plant, Nothapodytes nimmoniana. Endophytes, the microorganisms that reside within plants, are reported to have the ability to produce host-plant associated metabolites. Hence, our research aims to establish a sustainable and high camptothecin yielding endophyte, as an alternative source for commercial production of camptothecin. A total of 132 endophytic fungal strains were isolated from different plant parts (leaf, petiole, stem and bark) of $N$. nimmoniana, out of which 94 were found to produce camptothecin in suspension culture. Alternaria alstroemeriae (NCIM1408) and Alternaria burnsii (NCIM1409) demonstrated camptothecin yields up to $426.7 \pm 33.6 \mu \mathrm{g} / \mathrm{g}$ DW and $403.3 \pm 41.6 \mu \mathrm{g} / \mathrm{g} \mathrm{DW}$, respectively, the highest reported production to date. Unlike the reported product yield attenuation in endophytes with subculture in axenic state, Alternaria burnsii NCIM1409 could retain and sustain the production of camptothecin up to $200 \mu \mathrm{g} / \mathrm{g}$ even after 12 continuous subculture cycles. The camptothecin biosynthesis in Alternaria burnsii NCIM1409 was confirmed using ${ }^{13} \mathrm{C}$ carbon labelling (and cytotoxicity analysis on different cancer cell lines) and this strain can now be used to develop a sustainable bioprocess for in vitro production of camptothecin as an alternative to plant extraction.

Camptothecin, first reported in $1966^{1}$, is a monoterpene indole alkaloid. It is commercially produced from plants, mainly Camptotheca acuminata and Nothapodytes nimmoniana ${ }^{2}$. Camptothecin is the third most in-demand alkaloid after taxol and vinca-alkaloids for anti-cancer applications, and inhibits DNA topoisomerase I in cancer cells leading to cell death ${ }^{3}$. Among several camptothecin derivatives, topotecan, irinotecan and belotecan were approved and marketed as anti-cancer drugs, while others including silatecan, cositecan, lipotecan, simmitecan, chimmitecan, exatecan, namitecan, lurtotecan, elomtecan, diflomotecan, gimatecan, tenifatecan and genz-644282 are under various stages of clinical investigation ${ }^{4,5}$. Owing to the high demand of camptothecin, both the plants C. acuminata and N. nimmoniana are endangered and overharvested ${ }^{6}$. In the Western Ghats of India, complete trees of N. nimmoniana are uprooted for camptothecin extraction. To produce 1 ton of camptothecin, around 1000-1500 tons of $N$. nimmoniana wood chips are needed ${ }^{7}$. Hence, the annual demand for N. nimmoniana plants has been more than 1000 tons in recent years ${ }^{6}$. Thus, to prevent such plant sources from extinction and to meet the ever-increasing demand of camptothecin, an alternative means of camptothecin production is needed.

It is well known that the endophytes can have the potential to produce their host plant-based metabolites. Since the discovery of the first taxol producing endophyte ${ }^{8}$, endophytes in general have gained attention as a potent alternative source of plant metabolites. Though several successful attempts have been made to isolate endophytes, the commercial production of bioactive compounds using endophytes has not yet been established. The bottleneck has been the inconsistent product yield, which is generally reported to be lost with successive subculture under axenic state ${ }^{9}$. Many camptothecin producing endophytes have been reported in the last decade. However, the yields reported have been low $(0.5-45 \mu \mathrm{g} / \mathrm{g})$ and inconsistent with subculture in axenic state (Table 1).

${ }^{1}$ Department of Biotechnology, Bhupat and Jyoti Mehta School of Biosciences, Indian Institute of Technology Madras, Chennai 600 036, India. 'School of Ecology and Conservation, University of Agricultural Sciences, GKVK, Bangalore 560 065, India. ${ }^{3}$ Present address: JSS College for Women (Autonomous), Saraswathipuram, Mysore 570009, India. ${ }^{\square}$ email: smita@iitm.ac.in 


\begin{tabular}{|c|c|c|c|c|c|c|c|c|c|c|}
\hline \multirow[b]{2}{*}{ Endophytic fungus } & \multirow[b]{2}{*}{ Host plant } & \multicolumn{8}{|c|}{ Camptothecin yield of different generations } & \multirow[b]{2}{*}{ References } \\
\hline & & 1st & 2nd & 3rd & 4th & 5th & 6th & 7th & 8th & \\
\hline Fusarium solani INFU/CA/KF/3 & C. acuminata & $\sim 6^{\mathrm{a}}$ & $\sim 5.5^{\mathrm{a}}$ & $\sim 0.5^{\mathrm{a}}$ & $\sim 1^{\mathrm{a}}$ & $\sim 1^{\mathrm{a}}$ & $\sim 0.5^{\mathrm{a}}$ & $\sim 0.4^{\mathrm{a}}$ & & 2 \\
\hline Phomopsis sp. UAS014 & N. nimmoniana & + & $21.7^{\mathrm{a}}$ & $11.4^{\mathrm{a}}$ & $6.6^{\mathrm{a}}$ & & & & & 33 \\
\hline Aspergillus sp. LY341 & C. acuminata & $7.93^{\mathrm{b}}$ & $<\mathrm{LOD}^{*}$ & & & & & & & 21 \\
\hline Aspergillus sp. LY355 & C. acuminata & $42.92^{\mathrm{b}}$ & $4.06^{\mathrm{b}}$ & $<$ LOD $^{\#}$ & & & & & & 21 \\
\hline Trichoderma atrovirideLY357 & C. acuminata & $197.82^{\mathrm{b}}$ & $5.33^{\mathrm{b}}$ & $2.57^{\mathrm{b}}$ & $2.47^{\mathrm{b}}$ & $3.69^{\mathrm{b}}$ & $2.15^{\mathrm{b}}$ & $1.90^{\mathrm{b}}$ & $1.83^{\mathrm{b}}$ & 21 \\
\hline
\end{tabular}

Table 1. Reports on the attenuation of camptothecin produced from endophytes. ${ }^{\mathrm{a}} \mu \mathrm{g} \mathrm{g}{ }^{-1} ;{ }^{\mathrm{b}} \mu \mathrm{g} \mathrm{l}^{-1} ;{ }^{\#} L O D$ limit of detection.

\begin{tabular}{|c|c|c|c|c|c|c|c|}
\hline S. no & Explant & Endophytes & $\begin{array}{l}\text { Dried biomass obtained } \\
\text { from suspension cultures } \\
(\mathrm{g} / \mathrm{l})\end{array}$ & Camptothecin yield $(\mu \mathrm{g} / \mathrm{g})$ & Species full name & $\begin{array}{l}\text { Genbank accession } \\
\text { number }\end{array}$ & $\begin{array}{l}\text { NCIM catalogue } \\
\text { number }\end{array}$ \\
\hline 1 & Petiole & P4-6-PE2 & $6.2 \pm 0.1$ & $426.7 \pm 33.6$ & Alternaria alstroemeriae & MN795638 & NCIM1408 \\
\hline 2 & Leaf & P4-4-LE2 & $8.9 \pm 0.1$ & $403.3 \pm 41.6$ & Alternaria burnsii & MN795639 & NCIM1409 \\
\hline 3 & Leaf & P4-1-LE1 & $8.5 \pm 0.6$ & $269.4 \pm 53.9$ & Alternaria alstroemeriae & MN795640 & NCIM1441 \\
\hline 4 & Leaf & P5-4-LE1 & $9.8 \pm 0.2$ & $62.5 \pm 0.6$ & Alternaria angustiovoidea & MN795641 & NCIM1442 \\
\hline
\end{tabular}

Table 2. Highest yielding endophytes with their camptothecin yield, accession and deposition details.

In this study, we isolated a high camptothecin yielding endophyte from N. nimmoniana, demonstrating sustainable production of camptothecin in suspension culture. Increased relative abundance of ${ }^{13} \mathrm{C}$ labelled camptothecin molecules in the axenic culture extracts, when the fungus was cultivated in the presence of $\mathrm{D}_{-}\left[\mathrm{U}_{-}{ }^{13} \mathrm{C}\right]-$ glucose, demonstrated inherent biosynthesis of camptothecin in the isolated organism. Also, the cytotoxic effect of the crude extract of camptothecin from the fungal suspension culture was demonstrated on colon, lung, ovarian and breast cancer cell lines.

\section{Results}

Isolation of camptothecin yielding endophytes from Nothapodytes nimmoniana. Endophytes residing in the plants may vary with the type of plant tissue ${ }^{10,11}$. Therefore, leaves, petioles, stem and bark regions from Nothapodytes nimmoniana (J.Graham) Mabb. were used as explants for the comprehensive isolation of endophytes. Endophytes emerged from the cut ends of the explants after 5-7 days of incubation in potato dextrose agar medium (HiMedia, Mumbai). A total of 132 visually distinct endophytic strains were isolated from the explants of N. nimmoniana.

These isolated endophytes were then individually screened for their ability to produce camptothecin in suspension. Suspension cultures were initiated in duplicate and the biomass was harvested and dried for extraction of camptothecin for quantitative analysis using high performance liquid chromatography (HPLC). Among the 132 different endophytic isolates, 94 produced camptothecin in culture, with yields ranging from 0.1 to $400 \mu \mathrm{g} / \mathrm{g}$ DW (dry weight of biomass) (Supplementary Table S1).

Four of the highest yielding endophytes were selected for further analysis and their camptothecin yield was confirmed again in freshly initiated cultures (in triplicate). Camptothecin yields obtained from the suspension cultures of these strains are shown in Table 2. Strain P4-6-PE2, isolated from the petiole region of a young plant gave the highest yield of camptothecin in suspension $(426.7 \pm 33.6 \mu \mathrm{g} / \mathrm{g})$, followed by strain P4-4-LE2 isolated from the leaf region of the plant, with a yield of $403.3 \pm 41.6 \mu \mathrm{g} / \mathrm{g}$. It is to be noted that the yield of camptothecin obtained from the natural plant of C. acuminata varies from 0.5 to $2.5 \mathrm{mg} / \mathrm{g}^{12}$ and the yield from N. nimmoniana ranges from 0.8 to $3 \mathrm{mg} / \mathrm{g}^{13}$.

Camptothecin production was confirmed in the suspension cultures of these camptothecin yielding endophytic strains using tandem mass spectrometry (LC-MS/MS). As expected, a precursor mass to charge ratio $(\mathrm{m} / \mathrm{z})$ of 349.12 corresponding to that of camptothecin was observed in the authentic standard and in the extracts from all the shortlisted (top 4) endophytic strains (Fig. 1). Further, for structural confirmation, the precursor $\mathrm{m} / \mathrm{z}$ (349.12) was fragmented by applying collision energy of 35 electron Volts (eV) and an $\mathrm{m} / \mathrm{z}$ of 305.13 (fragment) after the loss of $\mathrm{CO}_{2}$ was witnessed (Supplementary Fig. S1 online), confirming the presence of camptothecin in the extract sample.

The presence of camptothecin in the endophytic extracts was confirmed using thin layer chromatography (TLC). Along with the camptothecin producing culture extract, a non-camptothecin producing culture extract was also spotted on the TLC plate for analysis. As shown in Supplementary Fig. S2 online, all four camptothecin yielding endophytic extracts gave spots corresponding to that of authentic camptothecin. In contrast, the spot was absent from a non-camptothecin producing strain.

Molecular characterization of the selected high camptothecin yielding endophytes. Endophytic strains grown on plates made with PDA medium showed difference in their morphological appearance 


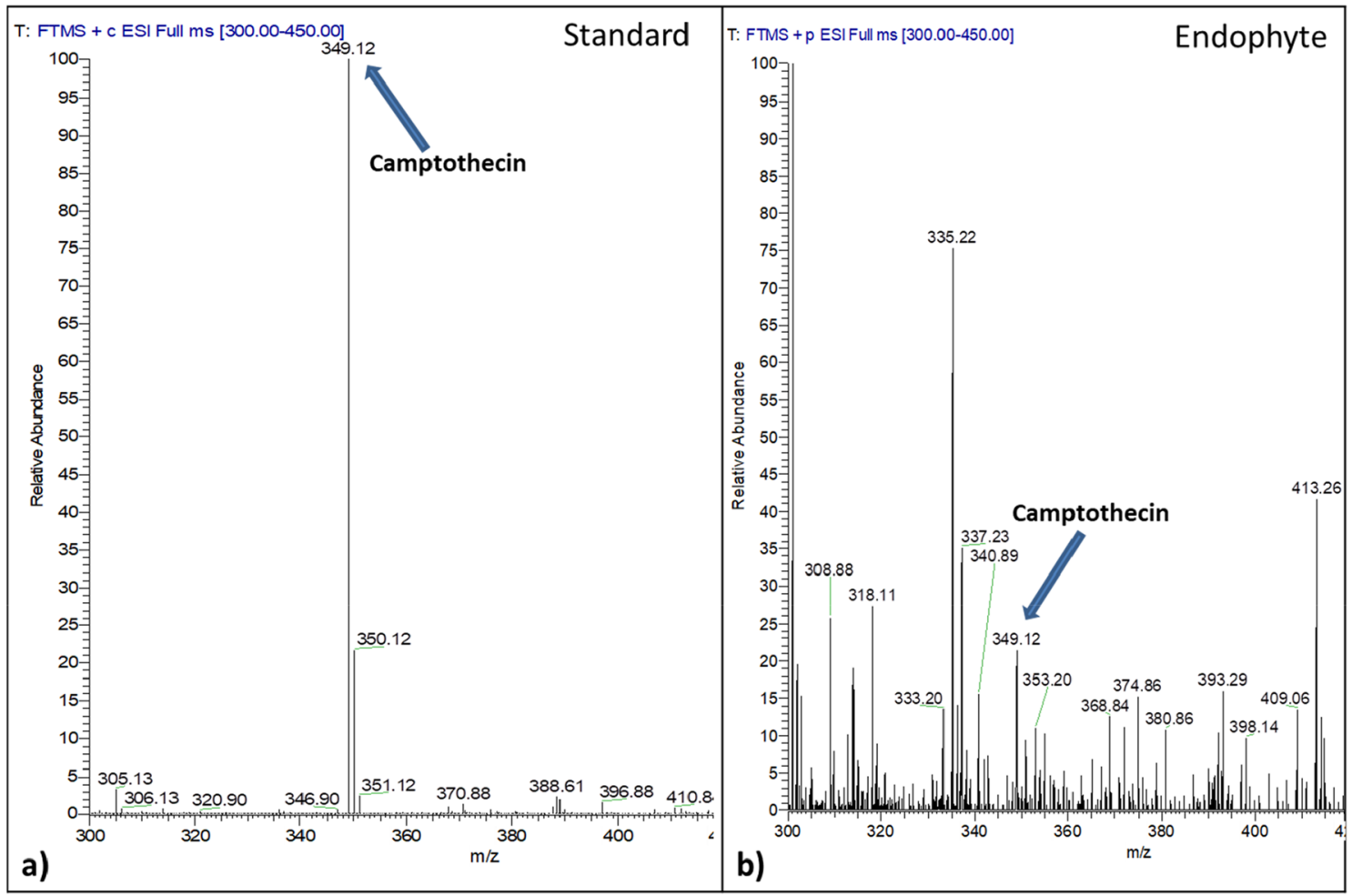

Figure 1. Identification of camptothecin from the endophytic extract using mass spectrometry. An $\mathrm{m} / \mathrm{z}$ of 349.12 corresponding to camptothecin visualized in both (a) the standard and (b) the isolated endophyte.

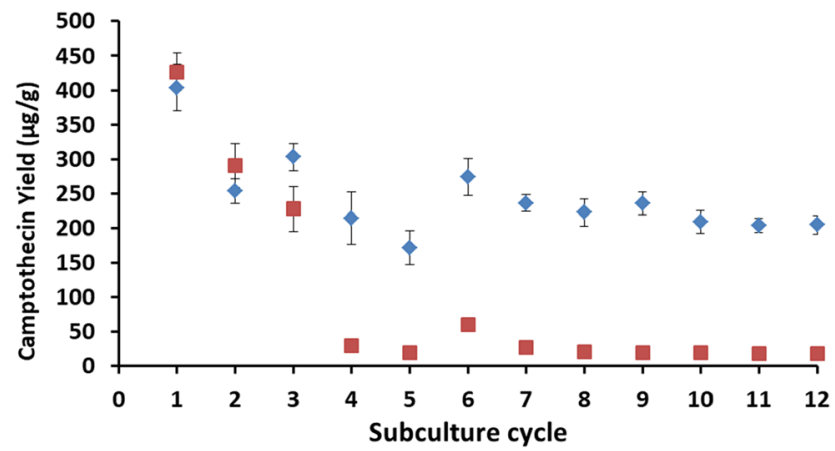

Figure 2. Stability analysis on camptothecin yield (average yield \pm SEM) from the two high yielding endophytic strains $(\diamond$ A. burnsii NCIM1409 and $\square$ A. alstroemeriae NCIM1408).

and all of them formed spores when viewed under a microscope (Supplementary Fig. S3 online). All four isolates were found to be different species of Alternaria genus. Among the four, the highest yielding strain was found to be A. alstroemeriae with a yield of $426.7 \pm 33.6 \mu \mathrm{g} / \mathrm{g}$ and the second highest was found to be $A$. burnsii with a yield of $403.3 \pm 41.6 \mu \mathrm{g} / \mathrm{g}$. Accession numbers from NCBI Genbank and the respective catalogue numbers of each strain deposited in NCIM have been listed in Table 2.

Sustainable production of camptothecin in the highest yielding endophyte. Sustainable production of camptothecin in the two highest camptothecin yielding endophytes was assessed in suspension culture generated from the 1 st through the 12 th generation slants. The highest yielding strain (A. alstroemeriae NCIM1408) demonstrated a sharp decrease in the camptothecin yield from $426.7 \pm 33.6 \mu \mathrm{g} / \mathrm{g}$ DW in culture developed from its 1 st generation slant to $17.9 \pm 0.7 \mu \mathrm{g} / \mathrm{g}$ DW in culture from its 12 th generation slant. This result was in accordance with the well-known product yield attenuation observed in axenic cultures of endophytes (Table 1). Interestingly, unlike the highest yielding strain, the second-highest yielding strain (A. burnsii NCIM1409) could demonstrate sustainable production of camptothecin (up to $\sim 200 \mu \mathrm{g} / \mathrm{g}$ ) in culture even from its 12th generation slant used as inoculum. As shown in Fig. 2, after an initial decrease in the yield to 
$254.1 \pm 17.9 \mu \mathrm{g} / \mathrm{g}$ DW in cultures developed from the second generation slant, this endophyte demonstrated a steady yield of $\sim 200 \mu \mathrm{g} / \mathrm{g}$ DW in cultures from 3rd till 12 th generation slant.

These results demonstrate that the highest yielding strain may not always be a sustainable producer, and it is equally important to consider sustainable production along with product yield of the metabolite during the screening and selection of a potential endophyte for successful bioprocess development.

Confirmation of camptothecin biosynthesis by $\boldsymbol{A}$. burnsii via carbon metabolism. Existence of host independent biosynthetic machinery in endophytes has been doubted due to product yield attenuation under an axenic state ${ }^{14}$. To confirm the endophytic strain has the endogenous ability to synthesize camptothecin, the cultures were fed with $\mathrm{D}_{-}\left[\mathrm{U}_{-}{ }^{13} \mathrm{C}\right]$-glucose and ${ }^{13} \mathrm{C}$ label incorporation into camptothecin was monitored. The fungal cultures fed with $\mathrm{D}$-glucose and $\mathrm{D}-\left[\mathrm{U}_{-}{ }^{13} \mathrm{C}\right]$-glucose showed similar growth and camptothecin production, evident from the biomass $(\sim 8 \mathrm{~g} / \mathrm{l} \mathrm{DW})$ and camptothecin yield $(\sim 200 \mu \mathrm{g} / \mathrm{g} \mathrm{DW})$ respectively, achieved after 8 days under similar cultivation conditions. An $\mathrm{m} / \mathrm{z}$ of 349.12 corresponds to that of camptothecin with all unlabelled carbon atoms and the presence of one ${ }^{13} \mathrm{C}$ labeled carbon would increase the molecular weight of camptothecin by 1 dalton. Since camptothecin has 20 carbons, there is a possibility that $1-20$ carbon atoms in the camptothecin molecule produced can get labelled in the crude extract of camptothecin when grown on $\mathrm{D}-\left[\mathrm{U}_{-}{ }^{13} \mathrm{C}\right]$-glucose. This was verified by tandem mass spectrometry confirming the increase in the molecular mass of camptothecin corresponding to the number of carbon atoms labelled in it. The mass spectrum revealed majority of carbon atoms (2-20) getting labelled in camptothecin. This was further supported by the MS-MS fragmentation pattern of the most abundantly labelled camptothecin molecules in the sample after the removal of labelled $\mathrm{CO}_{2}$ (Supplementary Table S2, Supplementary Fig. S4 online). Moreover, the absence of unlabelled camptothecin mass peak in $\mathrm{D}_{-}\left[\mathrm{U}_{-}{ }^{13} \mathrm{C}\right]$-glucose fed sample (Fig. $3 \mathrm{c}$ ) and absence of labelled camptothecin mass peak in D-glucose fed sample (Fig. 3a,b) could also substantiate labelled glucose metabolism and its incorporation in the synthesis of camptothecin by the organism.

Interestingly, the mass peak corresponding to camptothecin with 18 labelled carbons $\left({ }^{13} \mathrm{C}_{18} \mathrm{C}_{2} \mathrm{H}_{16} \mathrm{~N}_{2} \mathrm{O}_{4}\right)$ showed highest relative abundance (Fig. 3d; Supplementary Fig. S4 online) while $\mathrm{m} / \mathrm{z}$ corresponding to 20 labelled carbons $\left({ }^{13} \mathrm{C}_{20} \mathrm{H}_{16} \mathrm{~N}_{2} \mathrm{O}_{4}\right)$ in camptothecin was relatively less abundant. This was presumably due to the fact that potato infusion present in the medium is a source of unlabelled organic carbon which could have diluted the ${ }^{13} \mathrm{C}$ incorporation from $\mathrm{D}-\left[\mathrm{U}-{ }^{13} \mathrm{C}\right]$-glucose.

Cytotoxicity analysis of the camptothecin extract from $\boldsymbol{A}$. burnsii on cancer cell lines. As the source of camptothecin was novel, the cytotoxicity analysis was also carried out to further substantiate the potential commercial utilization of the microbial strain for various anti-cancer applications. The cytotoxicity of the crude extract of camptothecin from A. burnsii was tested on various cancer cell lines which includes human breast cancer (MCF7), human ovarian adenocarcinoma (SKOV3), human non-small cell lung carcinoma (H1299), colon adenocarcinoma (Caco-2, HT29), and non-cancerous embryonic kidney (HEK293T) cell lines. The $\mathrm{IC}_{50}$ values determined for the camptothecin extract and standard camptothecin against all the cancer cell lines studied have been shown in Table 3 as calculated from the plot of $\%$ cell viability vs. log concentration of the extract (Supplementary Fig. S5 online). Apart from MCF7, all other cancer cell lines showed a lower $\mathrm{IC}_{50}$ value for camptothecin extract from A. burnsii than the standard camptothecin ( $\geq 90 \%$ pure), which could be due to the effect of other metabolites present in the crude extract along with camptothecin (Table 3). Among all the cancer cell lines used, camptothecin extract from A. burnsii was found more toxic toward lung and colon cancer cells which is in accordance with the reports on derivatives of camptothecin ${ }^{4}$.

The cytotoxicity of the camptothecin extract from A. burnsii was further supported by clonogenic (Fig. 4) and wound healing assay (Fig. 5), where colony formation and healing were inhibited on the selected SKOV3 cell line.

\section{Discussion}

Extensive and invasive natural plant extraction of camptothecin to meet its increasing market demand, has endangered N. nimmoniana plant species in India ${ }^{6}$. Hence, an alternative method of its production is the need of the hour to prevent the natural plant source from extinction. Endophytes are being explored as one a potential alternative source for high-value plant metabolite production. A wide variation in the metabolite yield in plants due to climatic and geographical factors, slow growth rate of the plant and un-availability of the plant material in the wild, have been the key limitations to meet the market demand of camptothecin. On the contrary, a microbial fermentation process, which is amenable to optimization and scale-up for enhanced yield and productivity, can be an excellent alternative to the natural plant source for large-scale and sustainable production of camptothecin under in vitro conditions.

In this study endophytes were isolated from the plant parts including leaf, petiole, stem and bark regions as the distribution of endophytes in plants can vary with respect to plant tissue type. Also care was taken to surface sterilize the explants for endophyte isolation immediately after excision since the time between excision and isolation can reduce the viability of endophytes residing in the plant part ${ }^{15}$. Here, we have reported for the first time, a high camptothecin yielding endophyte which could demonstrate sustainable production of camptothecin in axenic state for more than twelve subculture cycles in liquid medium. Production of camptothecin was confirmed by various methods of qualitative and quantitative analysis, like HPLC, TLC and LC-MS/MS.

Though numerous endophytes are present in the plant, only a few of the isolated strains have the potential to produce the host-specific metabolite and many have shown attenuation upon subculturing 9,16 . Various factors were stated to play a role in the endophyte's non-sustainability, including their evolutionary relationship with the host plant ${ }^{17}$, and lack of host stimuli ${ }^{18}$ or gene silencing under axenic conditions ${ }^{19}$. Thus, comprehensive bioprospecting and screening are required to identify a sustainable and high product yielding strain of endophyte. In 


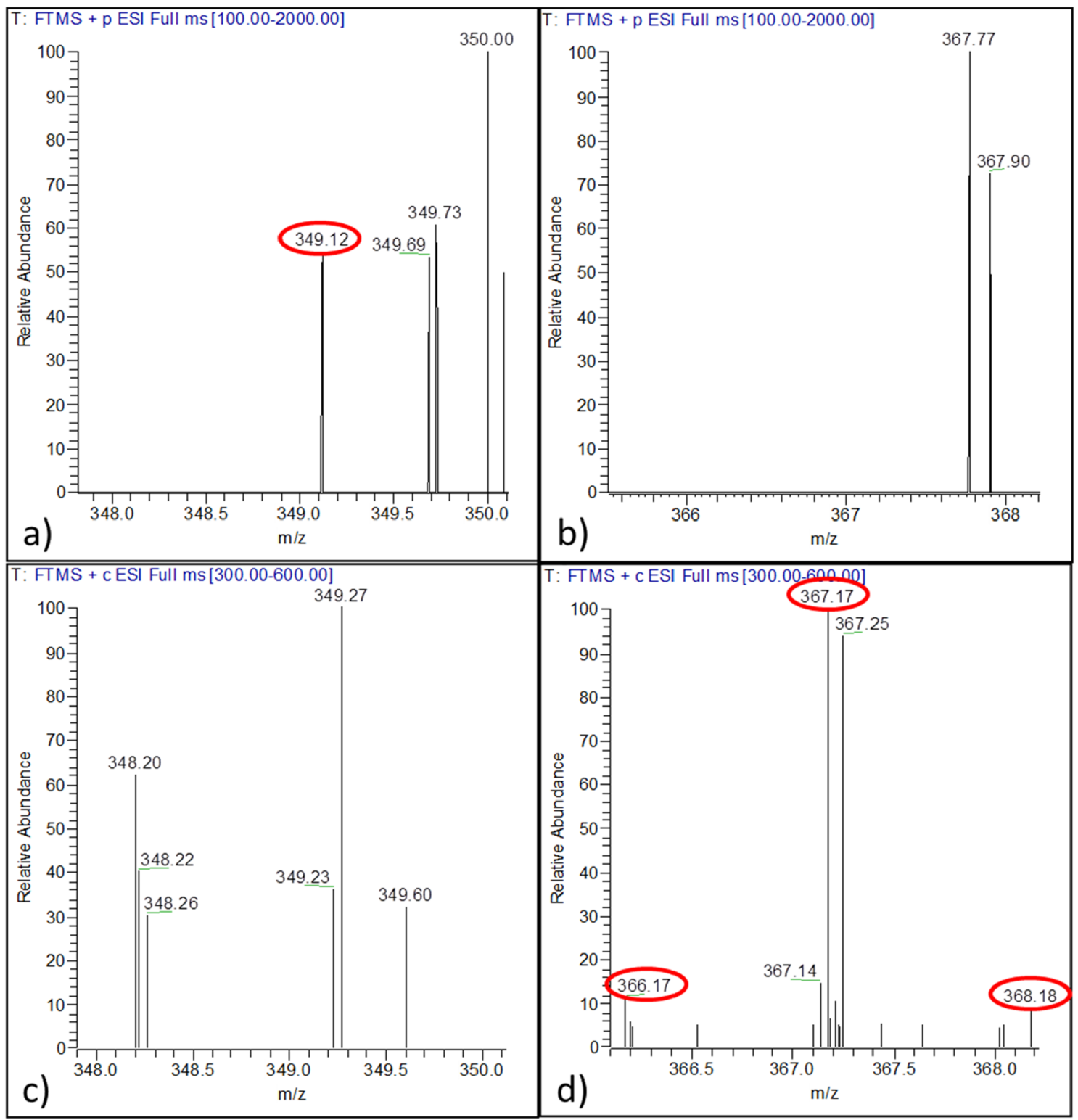

Figure 3. Confirmation of camptothecin biosynthesis in A. burnsii using D- $\left[\mathrm{U}_{-}{ }^{13} \mathrm{C}\right]$-glucose. (a) Mass spectrum of the crude extract of camptothecin shows that non labelled camptothecin $\mathrm{m} / \mathrm{z}$ of 349.12 was visualized only in D-glucose fed samples and (b) $\mathrm{m} / \mathrm{z}$ corresponding to labelled camptothecin was not present in them. (c) Non labelled camptothecin $\mathrm{m} / \mathrm{z}$ of 349.12 was not visualized in $\mathrm{D}-\left[\mathrm{U}-{ }^{13} \mathrm{C}\right]$-glucose fed cultures $(\mathbf{d}) \mathrm{an} \mathrm{m} / \mathrm{z}$ of 367.17 corresponding to 18 carbons in camptothecin being labelled was seen.

\begin{tabular}{|l|l|l|l|}
\hline Tissue & Cell Line & $\begin{array}{l}\mathbf{I C}_{\text {50 }} \text { value of camptothecin in the extract } \\
\text { of } \text {. burnsii }(\mathbf{n M})\end{array}$ & $\begin{array}{l}\mathbf{I}_{50} \text { value of camptothecin standard } \\
(\mathbf{n M})\end{array}$ \\
\hline Breast & MCF7 & $44.6 \pm 12.6^{* *}$ & $14.7 \pm 0.7$ \\
\hline Lung & H1299 & $13.6 \pm 1.7^{* *}$ & $93.3 \pm 22.6$ \\
\hline Ovary & SKOV3 & $117.4 \pm 10.4^{*}$ & $165.9 \pm 31.2$ \\
\hline Colon & HT29 & $50.1 \pm 11.2^{* * *}$ & $467.4 \pm 90$ \\
\hline Colon & Caco-2 & $26.9 \pm 3.5^{* * *}$ & $70.7 \pm 7.7$ \\
\hline Non-cancerous embryonic kidney & HEK293T & $281.5 \pm 33^{*}$ & $158.4 \pm 55.6$ \\
\hline
\end{tabular}

Table 3. Cytotoxic activity of crude extract of camptothecin from A. burnsii and camptothecin standard on the various cancerous and non-cancerous cell line. Confidence interval for statistically different $\mathrm{IC}_{50}$ of the camptothecin extract, in comparison to that of the standard, for each cancerous cell line: ${ }^{\star} \mathrm{p} \leq 0.05 ;{ }^{* *} \mathrm{p} \leq 0.01$; ${ }_{* * *} \mathrm{p} \leq 0.001$. 

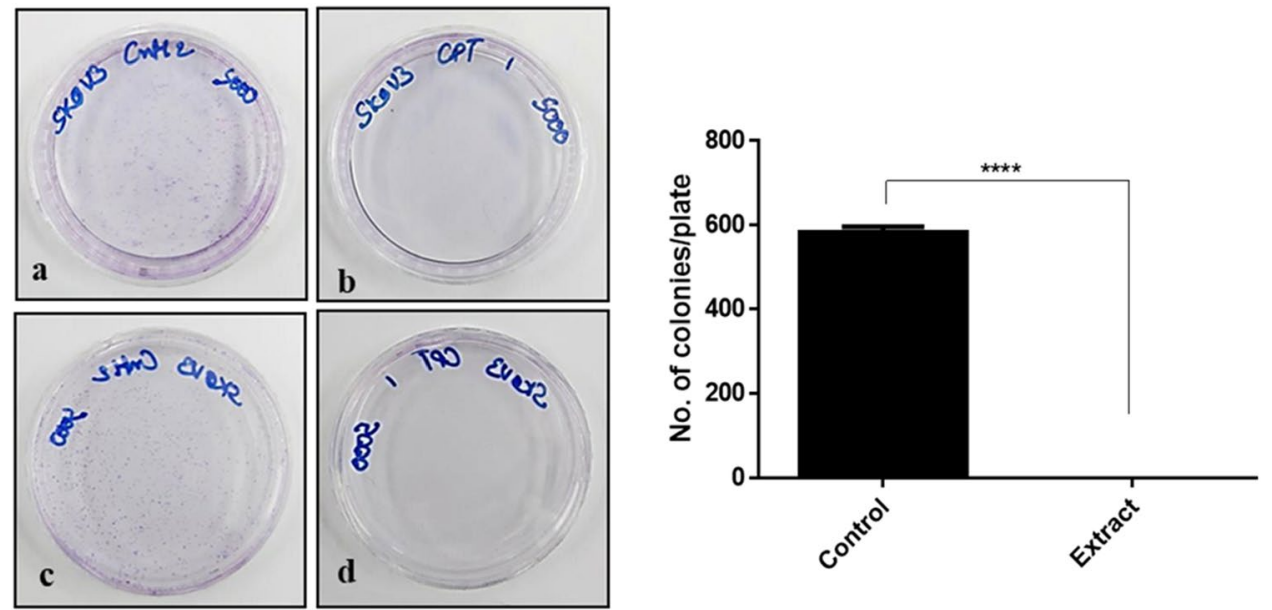

Figure 4. Complete inhibition of colony formation in the SKOV3 cell line by A. burnsii extract at $\mathrm{IC}_{25}$. SKOV3 control plates showed colony formation $(\mathbf{a}, \mathbf{c})$, whereas the treated plates showed no colony formation $(\mathbf{b}, \mathbf{d})$.
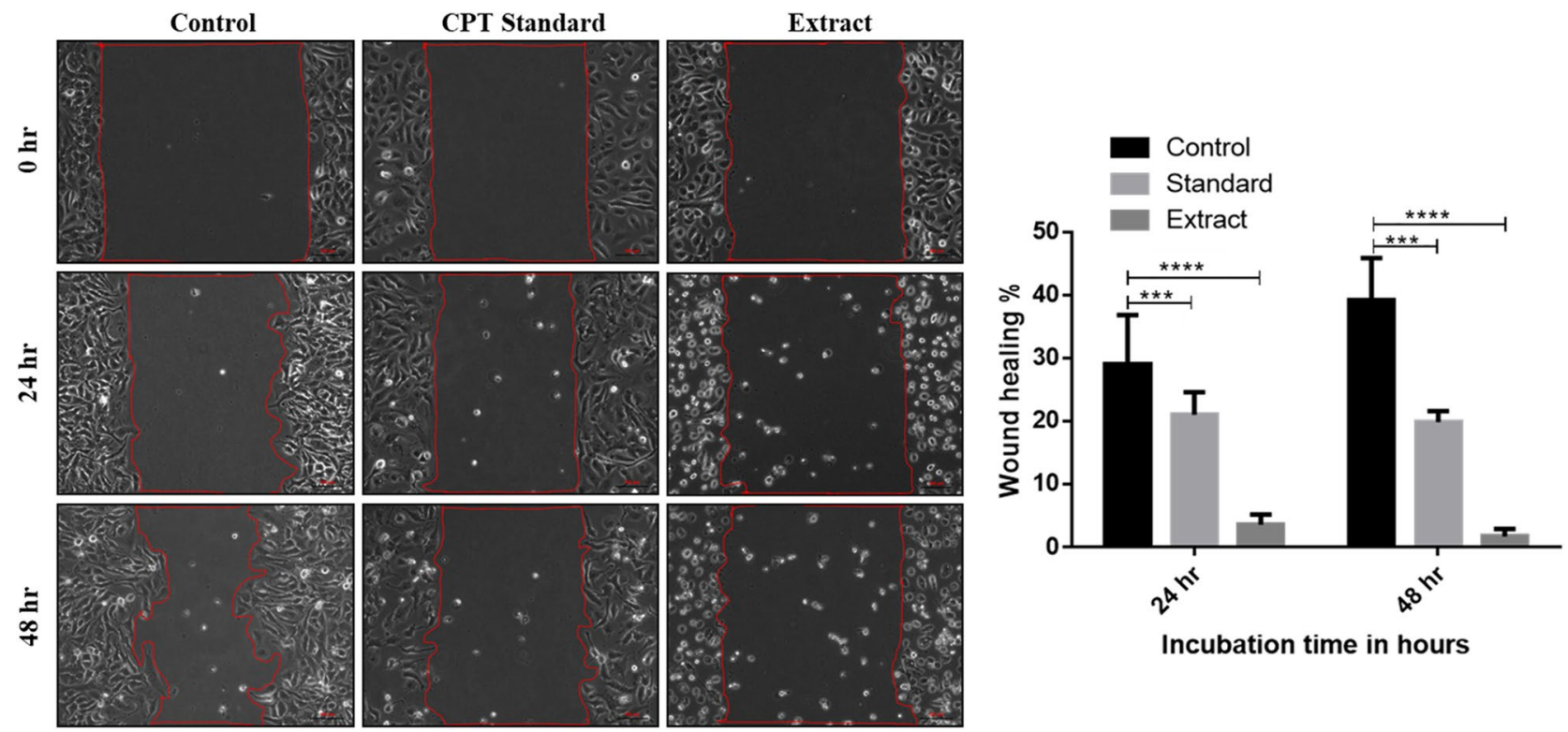

Incubation time in hours

Figure 5. Minimal wound healing observed with A. burnsii extract-treated SKOV3 cell line. Control cells showed $29 \%$ and $39 \%$ healing after 24 and $48 \mathrm{~h}$ respectively, while the standard camptothecin treated cells showed $20 \%$ and $19 \%$ healing after 24 and $48 \mathrm{~h}$ respectively. The crude extract-treated cells showed only $1 \%$ healing after $48 \mathrm{~h}$.

this study, sustainability in camptothecin production was tested in the highest yielding strains (A. alstroemeriae NCIM1408) and as expected the yield attenuated upon subsequent sub-culturing. However, in case of the second highest yielding strain, an initial decline in yield was observed and later the strain sustained the yield at $\sim 200 \mu \mathrm{g} / \mathrm{g}$ DW even after 12 subculture cycles. These results demonstrate that the highest yielding strain may not always be a sustainable producer, and it is equally important to consider sustainable production along with product yield of the metabolite during the screening and selection of a potential endophyte for successful bioprocess development.

Also the presence of host independent biosynthetic machinery in the endophytes was verified with the sustainable producing strain by feeding them with $\mathrm{D}-\left[\mathrm{U}_{-}{ }^{13} \mathrm{C}\right]$-glucose, as the endogenous potential of endophytes were often doubted ${ }^{14}$. Mass peaks corresponding to camptothecin with labelled carbons were detected in case of $\mathrm{D}$ - $\left[\mathrm{U}_{-}{ }^{13} \mathrm{C}\right]$-glucose fed samples, while the unlabelled camptothecin mass peak was absent in these samples, which confirmed the innate ability of the endophyte to produce camptothecin via its metabolic processes. This can be further supported by the literature evidence on enhancement and revival of attenuated camptothecin production upon media optimization ${ }^{20}$, elicitation ${ }^{21}$ and DNA methyltransferase treatment ${ }^{22}$. This indicates that potential of metabolite production in endophytes becomes cryptic under certain conditions and it can be restored upon alteration in the culture conditions. 
As the source of camptothecin was novel in the study, a cytotoxicity analysis of the crude extract of camptothecin was carried out on various cancerous and a non-cancerous cell lines. Interestingly, the extract was found to be more cytotoxic than the standard on most of the cell lines tested, which could be due to the synergistic effect of other compounds present in the crude extract ${ }^{23}$. The extract was also found to completely inhibit formation of colonies when clonogenic assay was performed with the selected cell line SKOV3 and just $1 \%$ healing after $48 \mathrm{~h}$ when wound healing assay was performed. This further substantiates the cytotoxicity of the extract on cancerous cell lines.

In conclusion, a high camptothecin yielding fungal endophyte A. burnsii NCIM1409 was isolated from N. nimmoniana which could demonstrate sustainable production of camptothecin under axenic conditions. Production of camptothecin by the microbial culture was verified via ${ }^{13} \mathrm{C}$ labeled glucose metabolism leading to labeled camptothecin biosynthesis. Interestingly, the crude camptothecin extract from the fungi demonstrated better cytotoxic activity (lower IC50) than the standard camptothecin ( $>90 \%$ purity) against the tested lung, colon and ovarian cancer cell lines and relatively less cytotoxicity against the non-cancerous cell line. The isolated strain can be used to develop a microbial-based sustainable bioprocess for large scale in vitro production of camptothecin for anti-cancer applications as an alternative to natural plant extraction.

\section{Methods}

Isolation of endophytes from Nothapodytes nimmoniana. Endophytes were isolated from 6 different plants of $N$. nimmoniana cultivated in the University of Agricultural Science, Bengaluru campus $\left(13.0762^{\circ}\right.$ $\mathrm{N}, 77.5753^{\circ} \mathrm{E}$ ). The plant specimen was authenticated as N. nimmoniana (J.Graham) Mabb. of the family Icacinaceae and deposited in Foundation for Revitalisation of Local Health Traditions (FRLH) herbarium, Bengaluru (FRLH 122013). The collected plant material (leaves, petioles, stem and bark) from young and mature plants was washed thoroughly in running tap water to remove the dust particles and then with sterile water. For surface sterilization of the explants, leaves and petioles were treated with $1 \%(\mathrm{v} / \mathrm{v})$ sodium hypochlorite for $1 \mathrm{~min}$ and then washed with sterile distilled water to remove sodium hypochlorite from the plant parts. The washed explants were then treated with $70 \%(\mathrm{v} / \mathrm{v})$ ethanol for $1 \mathrm{~min}$ and then washed again thoroughly with sterile distilled water to remove residual ethanol, if any, from the plant parts. This sterile water wash from the surface sterilized explants was streaked on a potato dextrose agar medium (in petri-plates) to check for the completion/ effectiveness of surface sterilization. Absence of any microbial growth on the said medium upon incubation even after 7 days confirmed the effectiveness/completion of surface sterilization. A similar procedure was carried out for the stem and bark regions, except that the treatment with $1 \%(\mathrm{v} / \mathrm{v})$ sodium hypochlorite was increased to $3 \mathrm{~min}$ and that with $70 \%(\mathrm{v} / \mathrm{v})$ ethanol was up to $5 \mathrm{~min}$. The sterile explants were then cut into small pieces $(\sim 1$ $\mathrm{cm}^{2}$ ) and placed on the petri dishes having PDA medium (HiMedia, Mumbai) supplemented with streptomycin $(100 \mu \mathrm{g} / \mathrm{ml})$ to prevent any bacterial growth, so as to preferentially isolate the endophytic fungi. The plates with explants were incubated at $28{ }^{\circ} \mathrm{C}$ in a biological oxygen demand (BOD) chamber for 7 days to allow the endophytes to emerge and grow from the cut ends. Morphologically distinct endophytes emerged from the cut ends after 5-7 days of incubation and were subsequently removed and plated separately on a fresh medium to obtain pure colonies of the endophytes.

A loop full of the mycelia was streaked on slants made with $5 \mathrm{ml}$ of potato dextrose agar medium (HiMedia, Mumbai) and incubated at $28^{\circ} \mathrm{C}$ for 7 days with an initial $\mathrm{pH}$ of 5.6. The slants were washed using $5 \mathrm{ml}$ of saline $(0.9 \%(\mathrm{w} / \mathrm{v}) \mathrm{NaCl})$ and were used as inoculum $(2 \% \mathrm{v} / \mathrm{v})$ for initiating suspension culture. Suspension cultures (in duplicate) were established with $50 \mathrm{ml}$ of potato dextrose broth (HiMedia, Mumbai) in $250 \mathrm{ml}$ Erlenmeyer flasks at an initial $\mathrm{pH}$ of 5.6. The suspension culture was allowed to grow in an incubator shaker at $28^{\circ} \mathrm{C}$ and $120 \mathrm{rpm}$. The shake flasks (in duplicate) were harvested after 8 days of the cultivation period for the estimation of biomass $(\mathrm{g} / \mathrm{l}, \mathrm{DW})$ and camptothecin yield $(\mu \mathrm{g} / \mathrm{g} \mathrm{DW})$ as per the protocol reported elsewhere ${ }^{23,24}$.

Biomass estimation. The biomass was harvested by centrifuging the cell suspension at $12,857 \times g$ for $15 \mathrm{~min}$ and discarding the supernatant. The pelleted cells were then washed with distilled water to remove traces of medium components and again separated by centrifugation at $12,857 \times \mathrm{g}$ for $15 \mathrm{~min}$. Washed biomass was then dried in a hot air oven at $60^{\circ} \mathrm{C}$ by spreading it on pre-weighed glass petri plates until constant dry weight was achieved.

Extraction of camptothecin. Camptothecin extraction from the fungal biomass was done as per the protocol reported earlier ${ }^{23}$. Briefly, the dried biomass $(0.3 \mathrm{~g})$ was dissolved in $20 \mathrm{ml}$ of distilled water and homogenized using a mortar and pestle, followed by liquid-liquid extraction, repeated thrice using $50 \mathrm{ml}$ of chloroform: methanol solvent mixture (4:1). The organic layer with extracted camptothecin was then collected and evaporated using a rotary evaporator. The dried camptothecin extract was re-suspended in $1 \mathrm{ml}$ of DMSO:methanol (1:50) and filtered through a $0.2 \mu \mathrm{m}$ filter for further analysis.

Quantification of camptothecin by HPLC. Twenty microliters of the camptothecin extract was injected in a reverse phase HPLC (LC-20AD, Shimadzu, Japan) at a flow rate of $0.8 \mathrm{ml} / \mathrm{min}$ using $25 \%$ acetonitrile as the mobile phase. ODS Hypersil gold column (Thermo Scientific) with a particle size of $5 \mu \mathrm{m}$ was used as the stationary phase at a column temperature of $30^{\circ} \mathrm{C}$. The absorbance of camptothecin was measured at $254 \mathrm{~nm}$ by a photodiode array detector ${ }^{25}$. Concentration of camptothecin in the sample was estimated using a standard calibration curve of peak area vs. known concentration of camptothecin (Supplementary Fig. S6 online), generated using authentic samples of camptothecin ( $>90 \%$ purity, Sigma Aldrich, MO, USA). The retention time of camptothecin from the standard was found to be $19.8 \mathrm{~min}$ and the area of the peak obtained from the endophytic 
extracts at the same retention time (Supplementary Fig. S7 online) was compared with the standard plot of camptothecin and the corresponding concentration and yield from the different fungal isolates were calculated.

Identification of camptothecin by LC-MS/MS. Camptothecin production in suspension culture of the high yielding isolates was further confirmed using tandem mass spectroscopy. This was based on qualitative identification of camptothecin, extracted from the harvested biomass, using an orbitrap elite mass spectrometer (ThermoScientific, Breman, Germany). A reverse-phase column (ODS Hypersil $\mathrm{C}_{18}$ column, $256 \times 4.5 \mathrm{~mm}, 5$ $\mu$ ) was connected to a heated electrospray ionization (H-ESI) source with the heater temperature maintained at $325^{\circ} \mathrm{C}$. The sheath gas flow rate was set at $40 \mathrm{arb}$, aux gas flow rate at 20 arb and sweep gas flow rate at 2 arb. The spray voltage for the H-ESI source was set at $4.5 \mathrm{kV}$ and the capillary temperature was maintained at $300^{\circ} \mathrm{C}$. MS grade water with $0.01 \%$ formic acid (solvent A) and acetonitrile (solvent B) in the ratio 3:1 was used as mobile phase at a flow rate of $500 \mu \mathrm{l} / \mathrm{min}$. Samples were injected into a $20 \mu \mathrm{l} \mathrm{sample} \mathrm{loop} \mathrm{and} \mathrm{analyzed} \mathrm{for} 60 \mathrm{~min}$ in the LC coupled with mass spectrometry set up in a mass scanning range of $300-450 \mathrm{~m} / \mathrm{z}$. The precursor ions were subjected to collision induced dissociation (CID) of $35 \mathrm{eV}$ to obtain fragment ions. Camptothecin, with a molecular formula of $\mathrm{C}_{20} \mathrm{H}_{16} \mathrm{~N}_{2} \mathrm{O}_{4}$, when analyzed in LC-MS under positive mode, yielded an intact mass $[\mathrm{M}+\mathrm{H}]$ of 349.12 . Further, for structural confirmation, by applying CID of $35 \mathrm{eV}$, the precursor $\mathrm{m} / \mathrm{z}(349.12)$ could be fragmented to an $\mathrm{m} / \mathrm{z}$ of 305.13 with a loss of $\mathrm{CO}_{2}{ }^{3}$.

Qualitative analysis of camptothecin by TLC. Presence of camptothecin in the endophytic extracts can also be identified using TLC. TLC was performed using silica gel $60 \mathrm{~F}_{254}$ aluminium plates (Merck, Germany). Extracts of camptothecin $(10 \mu \mathrm{l})$ from selected endophytes were spotted on the TLC plate with the standard camptothecin sample used as positive control. Chloroform-methanol (20:1) solvent system was used as the mobile phase. The plates were placed in the mobile phase in a pre-saturated chamber and the samples were allowed to be drawn upward on the TLC plates via capillary action for the separation of the sample components. As camptothecin is known to exhibit fluorescence under UV light, the TLC plates were dried and visualized at $254 \mathrm{~nm}$ in a UV chamber as per the protocol reported elsewhere ${ }^{26,27}$.

Molecular characterization of the selected endophytes. Freshly grown mycelia (200 mg) from the suspension cultures of the selected endophytes were taken and washed with ethanol before grinding to a fine powder with liquid nitrogen and sterile sea-sand in a mortar and pestle. The homogenized sample was then transferred into a sterile $1.5 \mathrm{ml}$ microcentrifuge tube and $200 \mu \mathrm{l}$ of cell-lysis buffer (Nucleo-pore DNA Sure Plant Mini Kit, Genetix Biotech, India) was added. This mixture was incubated at $70{ }^{\circ} \mathrm{C}$ for $10 \mathrm{~min}$ and then chloroform was added $(100 \mu \mathrm{l})$ and mixed thoroughly. Phase separation was then carried out in the microfuge tube after centrifugation at $20,000 \times \mathrm{g}$ for $15 \mathrm{~min}$. The top aqueous layer was separated into a sterile $1.5 \mathrm{ml}$ microcentrifuge tube for the isolation of DNA using nucleopore DNA sure plant mini-kit as per manufacturer's protocol (Genetix Biotech, India). Genomic DNA was isolated from the four highest yielding endophytic strains.

Isolated genomic DNA was amplified using internal transcribed spacer 1 (ITS1) (5' TCCGTAGGTGAACCT TGCGG $3^{\prime}$ ) and internal transcribed spacer 4 (ITS4) (5' TCCTCCGCTTATTGATATGC 3') primers ${ }^{28}$, along with the Phusion High Fidelity DNA polymerase (New England Biolabs, Ipswich). Touch-down method of PCR was used for increased specificity of primer amplification in a Surecycler 8800 (Agilent Technologies, Santa Clara, CA, USA). The upper limit of annealing temperature was set at $65^{\circ} \mathrm{C}$ and the lower limit at $53^{\circ} \mathrm{C}$. The PCR conditions used were as follows: initial denaturation for $5 \mathrm{~min}$ at $95^{\circ} \mathrm{C}, 35$ cycles of amplification including (1) denaturation at $95^{\circ} \mathrm{C}$ for $1 \mathrm{~min}$, (2) primer annealing to DNA at $65-53^{\circ} \mathrm{C}$ for $45 \mathrm{~s}$, (3) primer extension at $72^{\circ} \mathrm{C}$ for $2 \mathrm{~min}$ along with a final extension for $10 \mathrm{~min}$ at $72^{\circ} \mathrm{C}$. The purified polymerase chain reaction (PCR) products showed bands between 500 and $700 \mathrm{bp}$ corresponding to that of the conserved ITS regions in fungi. The amplified products were purified using a Qiaquick PCR purification kit as per manufacture's protocol (Qiagen, California, USA) and the size was confirmed using agarose gel electrophoresis. In the gel made with $0.8 \%(\mathrm{w} / \mathrm{v})$ agarose, $10 \mu \mathrm{l}$ of the amplified product and $2 \mu \mathrm{l}$ of $6 \mathrm{X}$ gel loading dye were added in the wells and the system was run at $50 \mathrm{~V}$ for $30 \mathrm{~min}$ before visualization of the amplicons on the gel under a UV transilluminator (GelDoc, BioRad, Italy). The purified PCR products were then sequenced by Sanger dideoxy method using AB 3130 Genetic Analyzer (Applied Biosystems, Foster City, CA, USA). Forward and reverse sequences were aligned to identify the consensus sequence consisting of ITS1, 5.8S and ITS4. These sequences were compared against the database of sequences using the nucleotide BLAST algorithm provided by (National Center for Biotechnology Information) NCBI. Also a phylogenetic tree (Supplementary Fig. S8 online) was constructed using maximum likelihood method based on Jukes-Cantor model $^{29}$ and the analysis was conducted in MEGA7 ${ }^{30}$. To increase the level of confidence in the taxonomic identifications, the comparisons were restricted to the sequences from the type material by checking the "sequences from type material" box in the general BLAST page ${ }^{31}$. The search results that displayed the highest query coverage and maximum score were used to determine the identity of the isolated high yielding endophytes. The sequences were deposited in NCBI and the strains were deposited in the National Collection of Industrial Microorganisms (NCIM) Pune, India.

Investigation of sustainable production of camptothecin in the endophytes. Sustainable production of camptothecin was investigated in the two highest yielding endophytes isolated in the study. Freshly isolated strains (stored as $25 \%$ glycerol stocks) were streaked separately as slants made of potato dextrose agar medium ( $5 \mathrm{ml}$ ) (HiMedia, Mumbai) and incubated at $28^{\circ} \mathrm{C}$ for 7 days with an initial $\mathrm{pH}$ of 5.6. These culture slants were used as inoculum for generating suspension and were considered as the first generation (subculture cycle) slants in the study. After 7 days, single mycelia from the first generation slant was picked and streaked on to the new slants with fresh medium ( $5 \mathrm{ml}$ of potato dextrose agar) under similar conditions. These were consid- 
ered as the second generation slants. Similarly, subsequent generation slants (up to 12th) were created after every 7 days of subculture on to fresh medium.

Suspension cultures from each generation slants were initiated as described earlier. After 7 days of growth, the slant-wash with $5 \mathrm{ml}$ of saline $(0.9 \% \mathrm{NaCl})$ was used as inoculum $(2 \% \mathrm{v} / \mathrm{v})$ for suspension culture. After 8 days of the cultivation period, the shake-flask cultures were harvested (in triplicate) for the estimation of biomass and camptothecin production.

Investigation of camptothecin biosynthesis using ${ }^{13} \mathrm{C}$ labelled glucose. In order to investigate and confirm camptothecin biosynthesis by the endophyte, suspension cultures were initiated using $20 \mathrm{~g} / \mathrm{l} \mathrm{D}-\left[\mathrm{U}_{-}{ }^{13} \mathrm{C}\right]-$ glucose (389374, Sigma Aldrich, MO, USA) as the carbon source in $50 \mathrm{ml}$ of sterilized potato infusion as growth medium in $250 \mathrm{ml}$ Erlenmeyer flasks with $2 \%(\mathrm{v} / \mathrm{v})$ of inoculum. The suspension cultures were allowed to grow in an incubator shaker at $28^{\circ} \mathrm{C}$ and $120 \mathrm{rpm}$ for 8 days of cultivation period before harvest (in duplicate) for the analysis of ${ }^{13} \mathrm{C}$-labelled camptothecin using LC-MS/MS.

For the preparation of potato infusion, $200 \mathrm{~g}$ of fresh potatoes were cleaned, peeled and chopped into smaller pieces and boiled for $15 \mathrm{~min}$ in $1000 \mathrm{ml}$ of distilled water at $90^{\circ} \mathrm{C}$. The liquid extract was then filtered through cheesecloth to be used as potato infusion. Extract from the culture grown only in potato infusion was used as a negative control to confirm that no camptothecin production was possible without added glucose in the medium.

Cytotoxicity analysis of extract on cancer cell lines by MTT assay. The crude extract of camptothecin from the selected endophyte A. burnsii was tested for its cytotoxic effect by performing 3-(4, 5-dimethylthiazol-2-yl)-2, 5-diphenyl tetrazolium bromide (MTT) (Sigma) assay on various cancer cell lines including MCF7, SKOV3, H1299, Caco-2, HT29, and HEK293T. All the cell lines were obtained from American Type Culture Collection (ATCC) and were maintained on Dulbecco's Modified Eagle Media (DMEM) supplemented with $10 \%$ fetal bovine serum (FBS) except SKOV3, which was maintained on McCoy's 5A (modified) medium supplemented with $10 \% \mathrm{FBS}$ at $37^{\circ} \mathrm{C}$ in an atmosphere of $5 \% \mathrm{CO}_{2}$.

In a 96 well plate, each well was seeded with 2500 cells in a volume of $100 \mu \mathrm{l}$ of their respective growth medium and incubated for $24 \mathrm{~h}$ after which varying concentration $(0.01-51.2 \mu \mathrm{M})$ of the crude extract of camptothecin and standard camptothecin were added, respectively to the medium. The cells were incubated for $72 \mathrm{~h}$ with the drug and then $0.5 \mathrm{mg} / \mathrm{ml}$ of MTT reagent in the respective medium was added and incubated for $5 \mathrm{~h}$. Then MTT reagent containing medium was removed and DMSO was added to each well after which the optical density was measured using a 96 well plate reader at a dual-wavelength of 570 and $650 \mathrm{~nm}$. MTT reagent forms formazan crystal within live cells, which then produces purple colour when dissolved in DMSO. The cell viability was calculated by comparing the optical density of the treated wells against the non-treated control wells.

$$
\text { Percentage cell viability }=\left(\frac{\text { Absorbance }_{570-650}(\text { Average of triplicate treated sample })}{\text { Absorbance }_{570-650}(\text { Average of triplicate control values })}\right) \times 100
$$

$\mathrm{IC}_{50}$ values were calculated using Graphpad Prism 6 (GraphPad Software, San Diego, CA) from the observed cell viability values and $\mathrm{SEM}^{32}$.

Clonogenic and wound healing assay. To investigate the inhibition of the colony formation of these cells in the presence of crude extract of camptothecin, clonogenic assay was performed. Viable cells (5000 per plate) were seeded and allowed to attach overnight in $60 \mathrm{~mm}$ plates. SKOV3 cells were incubated with the extract, at a concentration corresponding to their respective $\mathrm{IC}_{25}(58.7 \mathrm{nM})$, for 14 days in medium to facilitate colony formation. The obtained colonies were washed with PBS and fixed using acetic acid:methanol (3:1). The fixed cells were then stained with $0.2 \%(\mathrm{w} / \mathrm{v})$ crystal violet. The colonies formed were counted and compared with the DMSO controls in triplicate.

Similarly, a wound-healing assay was performed to study the inhibition of these cells' migration ability in the presence of the crude extract of camptothecin. SKOV3 cells were made to form a confluent monolayer on $60 \mathrm{~mm}$ plates by seeding 5 million cells on each plate. Using a $200 \mu$ micropipette tip, a wound or scratch was created on the confluent monolayer. The wounded cells were then rinsed with PBS and treated with the crude extract of camptothecin at a concentration corresponding to their $\mathrm{IC}_{25}(58.7 \mathrm{nM})$. The control plates were treated with an equal volume of DMSO. Using phase-contrast microscopy, the wound closure by cell migration was monitored and imaged at 0,24 and $48 \mathrm{~h}$ at the same spot. The wound area was calculated and the percentage of wound healing was compared between the drug-treated cells and DMSO treated cells as control ${ }^{32}$.

Statistical analysis. Statistical analyses were performed using Graphpad Prism 6 (GraphPad Software, San Diego, CA, USA). All experiments were performed in triplicates, and the results of MTT and clonogenic assay were analysed with $t$ test and that of wound healing assay was analysed with two-way ANOVA followed by a Dunnett's posthoc test. The values of ${ }^{\star} \mathrm{p}<0.05,{ }^{* *} \mathrm{p}<0.01,{ }^{* *} \mathrm{p}<0.001$ and ${ }^{\star * * *} \mathrm{p}<0.0001$ were considered as statistically significant.

Received: 4 November 2020; Accepted: 4 December 2020

Published online: 14 January 2021 


\section{References}

1. Wall, M. E. et al. Plant antitumor agents. I. The isolation and structure of camptothecin, a novel alkaloidal leukemia and tumor inhibitor from Camptotheca acuminata. J. Am. Chem. Soc. 88, 3888-3890 (1966).

2. Kusari, S., Zühlke, S. \& Spiteller, M. An endophytic fungus from Camptotheca acuminata that produces camptothecin and analogues. J. Nat. Prod. 72, 2-7 (2009).

3. Shweta, S. et al. Endophytic fungal strains of Fusarium solani, from Apodytes dimidiata E. Mey. ex Arn (Icacinaceae) produce camptothecin, 10-hydroxycamptothecin and 9-methoxycamptothecin. Phytochemistry 71, 117-122 (2010).

4. Pizzolato, J. F. \& Saltz, L. B. The camptothecins. Lancet 361, 2235-2242 (2003).

5. Musiol, R. An overview of quinoline as a privileged scaffold in cancer drug discovery. Expert Opin. Drug Discov. 12, 583-597 (2017).

6. Isah, T. \& Mujib, A. Camptothecin from Nothapodytes nimmoniana: Review on biotechnology applications. Acta Physiol. Plant. 37, 1-14 (2015).

7. Watase, I., Sudo, H., Yamazaki, M. \& Saito, K. Regeneration of transformed Ophiorrhiza pumila plants producing camptothecin. Plant Biotechnol. 21, 337-342 (2004).

8. Stierle, A., Strobel, G. \& Stierle, D. Taxol and taxane production by Taxomyces andreanae, an endophytic fungus of Pacific yew. Science 260, 214-216 (1993).

9. Kusari, S. \& Spiteller, M. Are we ready for industrial production of bioactive plant secondary metabolites utilizing endophytes?. Nat. Prod. Rep. 28, 1203-1207 (2011).

10. Collado, J., Platas, G., Gonzalez, I. \& Pelaez, F. Geographical and seasonal influences on the distribution of fungal endophytes in Quercus ilex. New Phytol. 144, 525-532 (1999).

11. Fisher, P. J., Petrini, O. \& Sutton, B. C. A comparative study of fungal endophytes in leaves, xylem and bark of Eucalyptus in Australia and England. Sydowia 45, 338-345 (1993).

12. Pi, Y. et al. Examination of camptothecin and 10-hydroxycamptothecin in Camptotheca acuminata plant and cell culture, and the affected yields under several cell culture treatments. Biocell 34, 139-143 (2010).

13. Padmanabha, B. V. et al. Patterns of accumulation of camptothecin, an anti-cancer alkaloid in Nothapodytes nimmoniana Graham, in the Western Ghats, India: Implications for identifying high-yielding sources of the alkaloid. Curr. Sci. 90, 95-100 (2006).

14. Venugopalan, A. \& Srivastava, S. Endophytes as in vitro production platforms of high value plant secondary metabolites. Biotechnol. Adv. 33, 873-887 (2015).

15. Golinska, P. et al. Endophytic actinobacteria of medicinal plants: Diversity and bioactivity. Antonie van Leeuwenhoek Int. J. Gen. Mol. Microbiol. 108, 267-289 (2015).

16. Deepika, V. B., Murali, T. S. \& Satyamoorthy, K. Modulation of genetic clusters for synthesis of bioactive molecules in fungal endophytes: A review. Microbiol. Res. 182, 125-140 (2016).

17. Zhao, J., Zhou, L., Wang, J. \& Shan, T. Endophytic fungi for producing bioactive compounds originally from their host plants. Curr. Res. Technol. Educ. Top. Appl. Microbiol. Microb. Biotechnol. 1, 567-576. https://doi.org/10.1016/j.phytochem.2012.07.021 (2010).

18. El-Elimat, T. et al. Flavonolignans from Aspergillus iizukae, a fungal endophyte of milk thistle (Silybum marianum). J. Nat. Prod. 77, 193-199 (2014).

19. Priti, V. et al. How promising are endophytic fungi as alternative sources of plant secondary metabolites?. Curr. Sci. 97, 477-478 (2009).

20. Clarance, P., Khusro, A., Lalitha, J., Sales, J. \& Paul, A. Optimization of camptothecin production and biomass yield from endophytic fungus Fusarium solani strain ATLOY-8. J. Appl. Pharm. Sci. 9, 35-46 (2019).

21. $\mathrm{Pu}, \mathrm{X}$. et al. Camptothecin-producing endophytic fungus Trichoderma atroviride LY357: Isolation, identification, and fermentation conditions optimization for camptothecin production. Appl. Microbiol. Biotechnol. 97, 9365-9375 (2013).

22. Vasanthakumari, M. M. et al. Restoration of camptothecine production in attenuated endophytic fungus on re-inoculation into host plant and treatment with DNA methyltransferase inhibitor. World J. Microbiol. Biotechnol. 31, 1629-1639 (2015).

23. Venugopalan, A., Potunuru, U. R., Dixit, M. \& Srivastava, S. Effect of fermentation parameters, elicitors and precursors on camptothecin production from the endophyte Fusarium solani. Bioresour. Technol. 206, 104-111 (2016).

24. Venugopalan, A. \& Srivastava, S. Enhanced camptothecin production by ethanol addition in the suspension culture of the endophyte, Fusarium solani. Bioresour. Technol. 188, 251-257 (2015).

25. Shweta, S., Gurumurthy, B. R., Ravikanth, G., Ramanan, U. S. \& Shivanna, M. B. Endophytic fungi from Miquelia dentata Bedd., produce the anti-cancer alkaloid, camptothecine. Phytomedicine 20, 337-342 (2013).

26. Rehman, S. et al. An endophytic Neurospora sp. from Nothapodytes foetida producing camptothecin. Appl. Biochem. Microbiol. 44, 203-209 (2008).

27. Lorence, A., Medina-Bolivar, F. \& Nessler, C. L. Camptothecin and 10-hydroxycamptothecin from Camptotheca acuminata hairy roots. Plant Cell Rep. 22, 437-441 (2004).

28. White, T. J., Bruns, T., Lee, S. \& Taylor, J. Amplification and direct sequencing of fungal ribosomal RNA genes for phylogenetics. In PCR Protocols: A Guide to Methods and Applications (eds Innis, M. A. et al.) 315-322 (Academic Press, New York, 1990).

29. Jukes, T. H. \& Cantor, C. R. Evolution of protein molecules. In Mammalian Protein Metabolism (ed. Munro, H. N.) 21-132 (Academic, New York, 1969).

30. Kumar, S., Stecher, G. \& Tamura, K. MEGA7: Molecular evolutionary genetics analysis version 7.0 for bigger datasets. Mol. Biol. Evol. 33, 1870-1874 (2016).

31. Raja, H. A., Miller, A. N., Pearce, C. J. \& Oberlies, N. H. Fungal identification using molecular tools: A primer for the natural products research community. J. Nat. Prod. 80, 756-770 (2017).

32. Tentu, S. et al. DHQZ-17, a potent inhibitor of the transcription factor HNF4A, suppresses tumorigenicity of head and neck squamous cell carcinoma in vivo. J. Cell. Physiol. 233, 2613-2628 (2018).

33. Gurudatt, P. S. et al. Attenuation of camptothecin production and negative relation between hyphal biomass and camptothecin content in endophytic fungal strains isolated from Nothapodytes nimmoniana Grahm (Icacinaceae). Curr. Sci. 98, 1006-1010 (2010).

\section{Acknowledgements}

The authors thank the Department of Biotechnology, Indian Institute of Technology Madras for the LC-MS facility and Department of Science and Technology (DST), Government of India for the financial assistance for research (Project number: EMR/2015/001418). The authors thank National Cancer Tissue Biobank (NCTB) facility at the Department of Biotechnology, Indian Institute of Technology Madras for, helping with the sequencing of the fungal endophytes.

\section{Author contributions}

K.M. isolated endophytes, designed and implemented the experiments, analyzed and compiled the results, formulated the manuscript. S.S. being the principal investigator conceptualized and designed the entire study, 
and contributed in data analysis and manuscript formulation. R.K. assisted in designing the experiments and analyzing the data for cytotoxicity analysis. S.K.R. provided the facilities and assisted and reviewed cytotoxicity analysis. K.S. assisted in collection of the plant material and in designing the experiment on isolation of endophytes. U.S. provided the facilities for isolation of endophytes and assisted and reviewed the data on the isolation of endophytes.

\section{Competing interests}

The authors declare no competing interests.

\section{Additional information}

Supplementary Information The online version contains supplementary material available at https://doi. org/10.1038/s41598-020-79239-5.

Correspondence and requests for materials should be addressed to S.S.

Reprints and permissions information is available at www.nature.com/reprints.

Publisher's note Springer Nature remains neutral with regard to jurisdictional claims in published maps and institutional affiliations.

(c) (i) Open Access This article is licensed under a Creative Commons Attribution 4.0 International License, which permits use, sharing, adaptation, distribution and reproduction in any medium or format, as long as you give appropriate credit to the original author(s) and the source, provide a link to the Creative Commons licence, and indicate if changes were made. The images or other third party material in this article are included in the article's Creative Commons licence, unless indicated otherwise in a credit line to the material. If material is not included in the article's Creative Commons licence and your intended use is not permitted by statutory regulation or exceeds the permitted use, you will need to obtain permission directly from the copyright holder. To view a copy of this licence, visit http://creativecommons.org/licenses/by/4.0/.

(C) The Author(s) 2021 